\title{
Technological development in Therapeutic applications of alternating electric fields: Review
}

\author{
Dr. S. Talele \\ The University of Waikato, New Zealand, sadhana@waikato.ac.nz
}

\begin{abstract}
A number of bacteria, virus and other unhealthy cells need to be killed for getting rid of them. For more than a century antibiotics have been effectively used for killing bacterial pathogens and chemical drugs against the cancer cells. However, there are bacteria and cancer cells that are drug resistant. This may have to be overcome by other stronger drugs, higher dosage. These can have detrimental side effects. Other non drug methods to aid the effect of these drugs have always been in research. Electrochemotherapy, a method of using electric fields along with the drug to be used topically has been one of the successful approaches. One of the most recent methods of Tumor Treating Frequencies (TTF) for a brain cancer has been FDA approved. This article details the use of TTF . The article also details some other latest research where alternating fields are used as antibacterial agents.
\end{abstract}

Index Terms - alternating electric field, biomedical applications.

\section{INTRODUCTION}

The 20th century was the modern era full of invention of antibacterial agents. Millions of people have been cured due to the treatment with the numerous antibiotic drugs used today. In the $21^{\text {st }}$ century these antibacterial drugs surely play an important role in the battle against the ever increasing variety of bacteria. However, the extensive use of antibiotics holds a threat in the future due to the rapid rise of multi drug resistant bacteria. Recently, a number of alternative modes like weak electric currents $[1,2,3,4]$, ultrasound wave therapy $[5,6,7]$, thermotherapy [8], photodynamic therapy [9] have been studied. These are expected to aid the antibiotics in the battle against bacterial pathogens. They definitely have an advantage of being non-drug based. However, the main drawback of the above mentioned methods is the high level of heating produced by the ultrasound waves and thermography [10]. In addition, the activated oxygen produced by the use of photodynamic therapy, both of which can damage the tissues in and surrounding the target area (11).

In addition, electric currents which are generated by the use of conductive electrodes are associated with the release of metal ions and free radicals at the electrode surface. These are well known to be toxic to living cells (12). As of today, none of the above-mentioned has been approved to be a method of treatment against bacterial control. However researchers have now shown that low intensity alternating fields of high frequencies have an in vitro inhibitory effect on growth of pathogenic bacteria.

\section{USE OF ALTERNATING FIELDS: BACKGROUND}

Alternating electric fields have been used since many years for the diagnosis, research and treatment of various medical conditions. The properties and effect of such electric fields differ, depending on their frequency and intensity. Very low frequencies (lower than $1 \mathrm{kHz}$ ) are capable of exciting the membrane of muscles and nerves, thus leading to membrane depolarization which leads to action potentials [13-15]. These have applications like fracture healing Defibrillators and bone growth.

Higher frequency alternating electric fields penetrate cells better, but the overall effect of hyperdepolarization on the cell membrane counteracts with polarization in a way that the integrated stimulation does not yield an action potential. However, at frequencies higher than $10 \mathrm{MHz}$, process of dielectric polarization eventually heats the tissue $[16,17]$.

These find use in Diathermy and radio frequency tumor ablation. Intermediate-frequency alternating electric fields, at frequencies between $10 \mathrm{kHz}$ and 1 $\mathrm{MHz}$, neither cause effective depolarization nor significant dielectric losses. Thus, these intermediate frequencies cannot stimulate muscles ad nerves, but also cannot seriously heat tissues at low enough 
intensities. Electric fields at these frequencies were considered to have no useful biological effect on cells $[16,18]$ until recent times when researchers have mentioned that these ac fields can be used more effectively in electroporation based biological applications $[19,20]$. These fields tend to be of higher intensity which can depend on the radius of the cell under investigation. Recently it has been found that such intermediate electric fields, have an effect that may lead to the death of dividing cells and thus named it as tumor treating fields (TTFields) [21,22].

The fields were found to have these properties already at a very low intensity $(<2 \mathrm{~V} / \mathrm{cm})$ and at intermediate frequency of $100--300 \mathrm{kHz}[21,22]$.

\section{FACTS ABOUT TUMOUR TREATING FIELDS:}

(a) TTFields's mechanism of action

Each cell contains numerous electrically charged molecules, such as proteins and DNA. When an alternating electric field is applied, these molecules will oscillate according to the changing direction of the field and its density. A uniform field will cause a movement parallel to the direction of the field. When the frequency of the field is high enough, such as in the case of TTFields, this molecular movement will reduce. Within a non-dividing cell, the field is mostly uniform and the net force on charges and dipoles will, therefore, yield minimal movement. Non-uniform electric fields cause electrophoresis forcing polar molecules to move toward higher field intensity [23, 24]. These high fields are at the furrow of a dividing cell called formed during mitosis. Such fields are characteristic of dividing cell when a narrow furrow connects the two forming daughter cells. Thus the electrical forces acting on the dividing cells affects in a way that mitosis becomes arrested for an abnormally long time [21]. It is reported that modelling results have shown that polarized molecules and organelles within the cell move toward the furrow due to the high dielectric force and disrupting the internal cell structure and cause the cell destruction [21].

(b) Other facts about Tumour Treating Frequency fields.

1. TTFields are generated by means electrically insulated ceramic electrodes, thus ensuring that no toxic ions are formed as there is no electrolysis [22] and can be effectively applied using surface electrodes [22].

2. TTFields can be delivered via a portable, light-weight $(\sim 3 \mathrm{~kg})$ device carried by the patient (NovoTTFields-100A, NovoCure Ltd, Haifa, Israel), connected to two pairs of insulated electrodes that can be applied to the patients' skin [21,22].

3. The device continuously (18 h/day on average) delivers two perpendicular $1-2 \mathrm{~V} / \mathrm{cm}$, $200 \mathrm{kHz}$ alternating electric fields [21,22].

4. The only treatment-related adverse event was mild-to-moderate contact dermatitis beneath the electrode gel, which was easily managed using topical treatments [21,22].

5. TTFields was shown to inhibit proliferation and to cause cell destruction of many cancer cells in vitro and in vivo [21,22].

6. There are no serious adverse events found related to TTFields as until now $[21,22]$.

7. Importantly, there were no cardiac or neurological abnormalities as a result of TTFields treatment $[21,22]$.

8. Due to non-invasive surface electrodes flow of ionic currents [25] or cell death [26] is prevented as compared to cell death as a result of direct currents, and thus continuous treatment is possible.

9. TTFields can actively inhibit different cell types, including multi-drug-resistant $[21,22]$.

10. TTFields does not need to be used along with any other therapy $[21,22]$.

11. Even though TTFields is a regional treatment, it still managed to decrease the likelihood of metastases formation in animal experiments [22].

TABLE I [27]

OPTIMAL FREQUNCY FOR DIFFERENT CELL LINES

\begin{tabular}{lc} 
Cell Line & $\begin{array}{c}\text { Optimal Frequency } \\
\text { in kHz }\end{array}$ \\
\hline B16F1 (mouse melanoma) & 120 \\
AA8 (Chinese hamster ovary) & 150 \\
VX-2 (rabbit kidney) & 150 \\
MCF-7 (human breast) & 150 \\
MDA-MB-231 (human breast) & 150 \\
F-98 (rat glioma) & 200 \\
U-87 (Human glioma) & 200 \\
U-118 (Human glioma) & 200
\end{tabular}




\section{FUTURE OF TTFIELDS}

This use of TTFields is one of the most recent researched non invasive, non drug method which is capable of inhibiting several cell types including multi drug resistant cell lines. Each cell line will be inhibited at an optimum frequency specific for the cell line [27] as shown in Table 1.With more research the treatment can be developed for further applications as follows:

1. There are also some data indicating that combining chemotherapeutic cancer treatments with TTFields may increase efficacy and sensitivity to chemotherapy [28].

2. TTFields is a regional treatment: it could be employed in situations where radiotherapy is not possible [27].

3. Even though TTFields is a regional treatment, it still manage to decrease the likelihood of metastases formation in animal experiments [29].

\section{ANTIBACTERIAL EFFECTS IF ALTERNATING FIELDS:}

Recent studies using alternating fields using insulated electrodes have reported to be useful [30]. Following are some facts about the use of antimicrobial fields (AMfields).

1. AMFields applied using noninvasive, insulated electrodes, have a significant inhibitory effect on the growth of a pulmonary bacterial infection in mice [31].

2. The inhibitory effect was observed when AMFields were applied either as a monotherapy or as an aid to antibiotic therapy [31].

3. Inhibition of bacterial growth is dependent on frequency of AMFields.ranging from $100 \mathrm{KHz}$ to $10 \mathrm{MHz}$ with different bacteria being sensitive to a specific frequency [31].

4. The antibacterial effect is also field intensity dependent with a $30 \%$ reduction reported at about $4 \mathrm{~V} / \mathrm{cm}$ [30] for $S$. aureus (strain SH1000).

5. The relatively high frequencies at which the AMFields effect is observed may be used for application of high intensities required for deep tissue treatment without nerve or muscle stimulation [30].
6. Future applications of AMFields can include treatment for resistant and/or chronic infections or to accelerate the treatment of common infections, such as tonsillitis, pharyngitis or similar, all by itself or in parallel with antibiotics [30].

\section{CONCLUSION:}

Alternating electric fields of intermediate frequencies when applied using non invasive electrically insulated electrodes have been reported to be beneficial in destroying some cancer cell lines and also have been found to have antimicrobial effects.

\section{References:}

[1] Caubet, R., F. Pedarros-Caubet, M. Chu, E. Freye, M. de Belem Rodrigues, J. M. Moreau, and W. J. Ellison. 2004. A radio frequency electric current enhances antibiotic efficacy against bacterial biofilms. Antimicrob. Agents Chemother. 48:4662-4664.

[2] Del Pozo, J. L., M. S. Rouse, G. Euba, C. I. Kang, J. N. Mandrekar, J. M. Steckelberg, and R. Patel. 2009. The electricidal effect is active in an experimental model of Staphylococcus epidermidis chronic foreign body osteomyelitis. Antimicrob. Agents Chemother. 53:40644068.

[3 ] Rabinovitch, C., and P. S. Stewart. 2006. Removal and inactivation of Staphylococcus epidermidis biofilms by electrolysis. Appl. Environ. Microbiol. 72:6364-6366.

[4] van der Borden, A. J., H. van der Werf, H. C. van der Mei, and H. J. Busscher. 2004. Electric currentinduced detachment of Staphylococcus epidermidis biofilms from surgical stainless steel. Appl. Environ. Microbiol.

70:6871-6874.

[5] Ensing, G. T., B. L. Roeder, J. L. Nelson, J. R. van Horn, H. C. van der Mei, H. J. Busscher, and W. G. Pitt. 2005. Effect of pulsed ultrasound in combination

with gentamicin on bacterial viability in biofilms on bone cements in vivo. J. Appl. Microbiol. 99:443-448.

[6] Rediske, A. M., N. Rapoport, and W. G. Pitt. 1999. Reducing bacterial resistance to antibiotics with ultrasound. Lett. Appl. Microbiol. 28:81-84 
[7] Pitt, W. G., M. O. McBride, J. K. Lunceford, R. J. Roper, and R. D. Sagers. 1994. Ultrasonic enhancement of antibiotic action on gram-negative bacteria.

Antimicrob. Agents Chemother. 38:2577-2582.

[8] Reithinger, R., M. Mohsen, M. Wahid, M. Bismullah, R. J. Quinnell, C. R. Davies, J. Kolaczinski, and J. R. David. 2005. Efficacy of thermotherapy to treat cutaneous leishmaniasis caused by Leishmania tropica in Kabul, Afghanistan: a randomized, controlled trial. Clin. Infect. Dis. 40:1148-1155.

[9] Jori, G., C. Fabris, M. Soncin, S. Ferro, O. Coppellotti, D. Dei, L. Fantetti, G. Chiti, and G. Roncucci. 2006. Photodynamic therapy in the treatment of microbial infections: basic principles and perspective applications. Lasers Surg. Med. 38:468-481.

[10] Zolfaghari, P. S., S. Packer, M. Singer, S. P. Nair, J. Bennett, C. Street, and M. Wilson. 2009. In vivo killing of Staphylococcus aureus using a lightactivated antimicrobial agent. BMC Microbiol. 9:27

[11] Oleinick, N. L., and H. H. Evans. 1998. The photobiology of photodynamic therapy: cellular targets and mechanisms. Radiat Res. 150:S146-S156.

[12] Liu, W. K., M. R. Brown, and T. S. Elliott. 1997. Mechanisms of the bactericidal activity of low amperage electric current (DC). J. Antimicrob. Chemother. 39:687-695.

[13] Polk, C. Therapeutic applications of low-frequency sinusoidal and pulsed electric and magnetic fields. In: Bronzino JD, editor, The biomedical engineering handbook. CRC Press, Inc., Boca Raton, FL; 1995. p. 1404-16

[14] Palti, Y. J. Stimulation of internal organs by means of externally applied electrodes. Appl Physiol 1966;21(5):1619-12

[15] Bassett, C.A. The development and application of pulsed electromagnetic fields (PEMFs) for ununited fractures and arthrodeses. Clin Plast Surg 1985;20:259-77

[16 ] Elson, E. Biologic effects of radiofrequency and microwave fields: in vivo and in vitro experimental results. In: Bronzino JD, editor, The biomedical engineering handbook. CRC Press, Inc., Boca Raton, FL; 1995. p. 1417-

[17] Chou, C. K. Radiofrequency hyperthermia in cancer therapy. In: Bronzino JD, editor. The biomedical engineering handbook. CRC Press, Inc., Boca Raton, FL; 1995. p. $1424-30$

[18] Goater, A. D, Pethig, R. Electrorotation and dielectrophoresis. Parasitology 1998;117(Suppl):S177-89
[19] C. Chen, J.A. Evans, M.P. Robinson, S.W. Smye, P. O'Toole, Measurement of the efficiency of cell membrane electroporation using pulsed ac fields, Phys. Med. Biol. 53 (2008) 4747-4757.

[20] Talele, S., P. Gaynor, J. van Ekeran, and M. J. Cree. Modelling single cell electroporation with bipolar pulse parameters and dynamic pore radii. Journal of Electrostatics, 68, pp. 261-274 (2010).

[21] Kirson, E. D, Gurvich, Z., Schneiderman, R., et al. Disruption of cancer cell replication by alternating electric fields. Cancer Res 2004;64(9):3288-95. TTFields significantly inhibited different cancer cell lines by disrupting cells undergoing mitosis.

[22] Kirson, E.D., Dbaly, V, Tovarys, F., et al. Alternating electric fields arrest cell proliferation in animal tumor models and human brain tumors. Proc Natl Acad Sci USA 2007;104(24):10152-7

[23] Clague, D.S., Wheeler, E.K., Dielectrophoretic manipulation of macromolecules: the electric field. Phys Rev E Stat Nonlin Soft Matter Phys 2001;64(2 Pt 2):026605

[24] Gonzalez, C.F., Remcho, V. T., Harnessing dielectric forces for separations of cells, fine particles and macromolecules. J Chromatogr A 2005;1079(1-2):59-68

[25] Webster, J.G., Clark, J. W., Medical Instrumentation: application and design. Wiley, New York; 1998

[26] Burnette, R. R., Ongpipattanakul, B. Characterization of the pore transport properties and tissue alteration of excised human skin during iontophoresis. J Pharm Sci 1988;77(2):132-7

[27] Miklos, Pless $\dagger$ \& Uri, Weinberg. Tumor treating fields: concept, evidence and future, Expert Opin. Investig. Drugs (2011) 20(8)

[28] Kirson, E. D., Schneiderman, R.S., Dbaly, V., et al. Chemotherapeutic treatment efficacy and sensitivity are increased by adjuvant alternating electric fields (TTFields). BMC Med Phys 2009;9:1

[29] Kirson, E. D., Giladi, M., Gurvich, Z., et al. Alternating electric fields (TTFields) inhibit metastatic spread of solid tumors to the lungs. Clin Exp Metastasis 2009;26(7):633-40

[30] Giladi, M., Y. Porat, A. Blatt, Y. Wasserman, E. D. Kirson, E. Dekel, and Y. Palti. 2008. Microbial growth inhibition by alternating electric fields. Antimicrob. Agents Chemother. 52:3517-3522. 
Infection, Antimicrob. Agents Chemother. Aug. 2010, p.

[31] Moshe Giladi, Yaara Porat, Alexandra Blatt, Esther $3212-3218$

Shmueli, Yoram Wasserman, Eilon D. Kirson, and

Yoram Palti, Microbial Growth Inhibition by Alternating

Electric Fields in Mice with Pseudomonas aeruginosa Lung 
This document was created with Win2PDF available at http://www.win2pdf.com. The unregistered version of Win2PDF is for evaluation or non-commercial use only. This page will not be added after purchasing Win2PDF. 